\title{
Editorial
}

\section{The Lost Art of Clinical Skills}

(BIRDEM Med J 2019; 9(2): 102-105)

\section{Introduction}

Sir William Osler told "Medicine is learned at bed side, not in classroom". For clinical diagnosis, history contribute major (60\%), physical examination- $25 \%$ and investigation- $15 \%$. So, there is no alternative to clinical skill.

This scenario is changing. With the rapid extension of laboratory tests of greater accuracy, there is a tendency for some clinicians and hence for some students in reaching a diagnosis to rely more on laboratory reports. They depend less on the history of the illness, the examination and behavior of the patient and clinical judgment. While in many cases laboratory findings are invaluable for reaching correct conclusions, the student should never be allowed to forget that it takes a man/ woman, not a machine, to understand a man/woman.

By the end of World War II, x-rays, which formerly entailed several minutes of exposure time, could be performed in a matter of seconds and for the first time, arterial blood gas measurement was possible. As attractive as these technologies seemed in the 1940s, the advancements of medical technology since then are even more alluring. Yet, technology seems to be replacing basic medical skills rather than complementing them. In "Introduction to Clinical Medicine: A Time for Consensus and Integration" Omori and colleagues discuss concerns over historytaking and physical examination instruction, particularly during the first 2 years of medical school. ${ }^{1}$ It was decided in 2004 during introduction of USMLE step 2.The authors recommend a national collaboration on the integration of clinical skills education through medical school curriculum. Although this is a critical initial step, beyond the poor acquisition of basic clinical skills is the documented decline of some rudimentary skills after the second year of medical school. Why should history taking and physical examination skills remain esoteric concepts and plateau or decrease when they should be used in actual practice of medicine?
Regardless of the formal educational curriculum, the skills should improve if they are used to guide patient care decisions.

\section{Evidences}

Multiple editorials have decried the loss of clinical skills and medical educators justify not only teaching but also using basic history-taking and physical examination skills in routine patient care. However, some skeptics suggest that emphasis on clinical skills is from a bygone era and that the availability of advanced imaging techniques and laboratory tests have supplanted ambiguous history and physical findings. Numerous studies and meta-analyses describe the poor operating characteristics of most history-taking and physical examination findings. For example, in a meta-analysis identifying the symptoms, signs, and tests most useful in diagnosing congestive heart failure (CHF), no single history-taking or physical examination findings provided adequate discrimination. The most discerning features of $\mathrm{CHF}$ - such as the presence of paroxysmal nocturnal dyspnea, an S3 gallop, or jugular venous distention - have such a low incidence (each documented in $50 \%$ of patients with $\mathrm{CHF}$ ) that their presence is not particularly helpful in diagnosing CHF. In fact, the best single predictor of CHF was found to be a B-type natriuretic peptide (BNP) value of 250 $\mathrm{pg} / \mathrm{mL}$ with a sensitivity of $89 \%$ and a specificity of $81 \%$. However, physicians rarely make decisions based upon one isolated finding, and the overall gestalt of the patient's illness is perhaps most important. In the CHF study, a high initial clinical suspicion (based upon the complete history and physical) was the most predictive element in the diagnosis of CHF. In fact, the authors concluded that ordering a BNP level was useful only in cases with an equivocal clinical suspicion. Instance of clinical judgment was even more apparent in a study of patients presenting to an outpatient clinic with either abdominal pain or chest pain. Physicians correctly classified $93 \%$ of patients with abdominal pain and $98 \%$ of patients with chest pain as having either an organic or nonorganic etiology solely on the basis of their initial 
clinical judgment and before ordering any diagnostic tests. These findings clearly indicate that clinical skills are important, but can technology replace thorough history-taking and physical examinations? Contrary to the paramount importance often ascribed to technology, numerous studies have demonstrated that technology has not necessarily improved the quality of patient care. Successive cohorts of autopsies over the last century have consistently demonstrated similar rates of misdiagnosis despite the use of advanced diagnostic procedures. For example, unknown malignancies were diagnosed in $36.5 \%$ of autopsies in 1923, compared with $41 \%$ in 1972 and $44 \%$ in 1998. In another study of 167 patients who died during a stay in the intensive care unit, autopsies uncovered a major diagnostic error in $32 \%$ of patients despite extensive diagnostic testing. In fact, the use of imaging was noted to contribute to misdiagnosis. Physicians missed the diagnosis of endocarditis in 9 patients despite ordering echocardiograms in the week before each of their deaths. By contrast, 3 patients diagnosed with endocarditis by echocardiogram subsequently had their diagnoses refuted during autopsy. In another autopsy study, the authors determined that ultrasound and computed tomography scanning provided misleading information for $7 \%$ of patients, whereas history and physical examinations rarely misled physicians ( $1 \%$ for history and $2 \%$ for physical examination). More recently, the Medicare program evaluated the use of imaging services nationwide and found a threefold variation in the number of examinations obtained across the United States. Despite substantial differences in the use of imaging studies, no demonstrable changes in quality or patient outcomes were noted. Although recent technological advances offer numerous tools to aid in diagnosis, their use should be guided by thorough history and physical examinations. The aforementioned study of autopsy results concluded that history-taking and physical examinations provided conclusive information for determining the main diagnosis in $73 \%$ and $62 \%$ of patients, respectively. Studies have consistently demonstrated that history-taking and physical examinations are the most important factors in arriving at a correct diagnosis, whereas lab tests and imaging studies play only minor roles.

\section{Discussion}

By comparison, imaging techniques provided conclusive information for diagnoses of $35 \%$ of patients and standard lab tests for $22 \%$ of patients. In fact, inaccurate, incomplete, or misinterpreted patient histories are among the leading causes for diagnostic errors. Physicians acknowledge the poor discrimination of individual history and physical examination findings but often neglect to consider the true sensitivity and specificity of imaging and other tests. Using technology becomes a "gold standard" for diagnosis instead of an adjunct to clinical judgment.

The state of clinical skills: Inconsistencies between laboratory findings and clinical data go undetected simply because too many physicians are insufficiently disciplined in the proper use of clinical skills and in the analysis of clinical data. Too often, palpably illogical laboratory findings are accepted without question. Despite the importance of history-taking and physical examination, clinical skills education has decreased since the 1960s, with deficiencies beginning in medical school and continuing through residency and into practice. With the erosion of thorough history taking and physical examinations, clinical reasoning (the ability to develop the gestalt impression) has also decreased. Although essential to determining the correct diagnosis, appropriate history-taking and physical examination are futile without the ability to interpret gathered information. History-taking is frequently limited by close-ended questions that fail to gather specific details critical to clinical decision-making. For example, instead of determining the severity, context, alleviating factors, exacerbating factors and chronology of dyspnea, its presence becomes a yes or no response. Beyond the clear decline in skills, medical students who demonstrate proper technical ability have serious deficiencies in clinical reasoning before they enter residency training. In a study comparing student scores on an objective structured clinical examination (OSCE), which required a complete organ system examination and a clinical performance examination (CPX), which required a focused physical based on the patient's history, a large discrepancy was noted among student scores. Students who excelled in the technical examination (OSCE) performed inconsistently when deciding on the appropriate physical examination elements during the patient scenario (CPX). In fact, the two scores showed 
no correlation on individual cases. Apparently, being able to perform a physical examination correctly during an OSCE (the standard used by most medical schools) does not translate into appropriate use of those skills in patient care.

Clinical skills in teaching hospitals: A glimpse of clinical education during medical school and residency illuminates several reasons for the overall decline in clinical decision-making skills. Before 1970s, internal medicine rounds with the attending physician were considered the platform for demonstrating historytaking, physical examinations, and clinical decisionmaking. Attending physicians demonstrated clinical skills, refined a physician-in-training's techniques, and corrected errors or misinterpretations. By 1978, the rate of performing bedside examinations during teaching time had decreased to $16 \%$, and many physicians estimate that number has decreased even further. Today, physicians-in-training rarely see attending physicians demonstrate history-taking and physical examination techniques and apply their findings to clinical decisionmaking. Rounds at many hospitals have shifted from bedside interactions with patients focused on the patient's history and physical examination to conference room learning focused on the patient's latest laboratory and imaging results. A recent national survey of medical students inquired about the quality of teaching during the inpatient component of their internal medicine clerkships. One third of students reported that their attendings rarely or never saw new patients with the team, not including the number who saw but did not examine the patients with the team. Not only are senior physicians failing to demonstrate clinical skills, but physicians-in-training are rarely observed taking histories or performing physical examinations, or offered feedback on their clinical skills. Less than $50 \%$ of medical students reported that the attending physician observed them performing some aspect of a physical examination, and less than $20 \%$ reported they were observed interviewing a patient. Even when faculty members do observe a student's performance, many do not identify the errors made by those students during their examinations. Observation and feedback are essential because physicians-in-training commit a high number of errors in routine history-taking and physical examinations. These errors have significant consequences; in one study, an attending physician's physical examination ultimately changed the diagnosis or disease management for one-quarter of the patients admitted to an internal medicine service, providing proof that any feedback on clinical skills is beneficial. In another study of the accuracy of resident presentations, resident physical examination skills directly correlated to the amount of time attending physicians spent with them at the bedside examining patients. However, physical examination skills did not correlate with classic measures of medical knowledge, such as resident intraining examination scores or prior medical school class rank, indicating those measurements do not automatically translate to clinical skills. Despite its decline, both medical educators and physicians-intraining still consider bedside teaching one of the most valuable learning tools. Although many lament the changes in medical education focus, medical schools overall have done little to improve the way clinical skills are taught. Since the 1980s, innovations in medical education have attempted to refocus the activities of medical schools and teaching hospitals back on education. New modalities, such as standardized patients, simulators, and computer technology, have been included to improve the teaching of clinical skills. However, these recent instructional methods have one common factor - they require less faculty involvement. In a recent case study, faculty members at one medical school consistently made curricular decisions that would best preserve faculty research time, resulting in a persistent decrease over the last half-century in studentfaculty contact. Identifying faculty who will take the time to teach medical students is now one of the most substantial difficulties faced by course directors. Although many of the new teaching technologies offer outstanding, nonintimidating platforms for students to refine their skills, they cannot replace patient interactions supervised by faculty.

\section{Conclusion}

Current movements to improve clinical skills education are essential and long overdue. Acknowledging that the provision of high quality medical care requires strong clinical skills and clinical reasoning, the Association of American Medical Colleges established the Task Force on the Clinical Skill Education of Medical Students. Several recommendations from this task force emphasize the importance of clinical skills. First, the task force acknowledged that clinical skills education 
is a longitudinal process that must be taught throughout medical school, residency training, and postgraduate work. Clinical skills are often ignored after the second year of medical school, even though they should be continuously reinforced and advanced to include more sophisticated techniques. Second, the task force emphasized that faculty members must take the primary role in teaching clinical skills. The development of clinical skills requires close mentorship with someone who can not only teach the specified skills, but also assess and provide feedback to the student. Third, the task force recommended that the evaluation of clinical skills must be patient-centered. Simulators and computerized technology are useful adjuncts to teaching clinical skills, but to deliver high-quality patient care, students must learn with actual patients. Emphasizing clinical skills is as significant as acknowledging the impact of the hidden curriculum perpetuated in many clinical arenas. Students and residents will value what their faculty mentors value and their observations of faculty-patient interactions demonstrate what skills are truly important to succeed as a physician. Rushed rounds with attending physicians exaggerate the importance of ancillary testing at the expense of clinical skills. Physician educators must not only dedicate themselves to teaching clinical skills, but also emulating those skills in everyday work. They must integrate new technology into clinical education without diverting attention from patients. Expectations for physicians-in-training should include a progressive improvement in clinical skills, guided by faculty feedback to correct and advance those skills. Although clinical faculty members play an essential role in promoting the development of necessary clinical skills in physicians-in-training, they must have sufficient support from medical school or teaching hospital leadership, as well as individual departments.

Barriers :Even motivated teaching faculty find numerous barriers within the administration of the medical school or teaching hospital. Medical education, particularly basic clinical skills education, is a low priority, especially in comparison to the incomegenerating endeavors of research and clinical work. In general, faculty are given little time to dedicate to teaching duties, so they either risk salary cuts or they teach "on their own time" after completing their clinical or research activities. At one medical school, it was estimated that, after paying for fringe benefits, faculty members were compensated approximately $\$ 16$ for each hour they spent teaching. In addition, teaching activities often do not contribute significantly to promotion and tenure decisions. Many medical school promotion and tenure committees have difficulty accepting the expanded definition of scholarship that rewards certain teaching activities. Ultimately, medical schools and teaching hospitals must instill value in teaching basic clinical skills by providing physicians the time and the compensation for these activities. Teaching clinical skills is time-intensive and requires dedicated faculty who are able to demonstrate, teach, and provide feedback. Current faculty members may lack the clinical expertise to be successful, adding to the difficulty in finding strong physician models proficient in teaching clinical skills. Most current medical school or teaching hospital faculty completed their education during a time of diminished emphasis on clinical skills, so they may lack confidence in their own skills. Teaching is not an intuitive endeavor for all physicians, and faculty are limited in their ability to assess clinical skills and provide quality feedback to physicians-in-training.

Faculty development is necessary to improve the current clinical skills of medical school faculty and provide proper instruction on the teaching and evaluation of clinical skills. Although history-taking and physical examination skills are often considered rudimentary, they serve as the foundation for all clinical decisionmaking and their significance should not be disregarded or forgotten. Modern technology has improved physician understanding of ailments and created new tools to use in diagnostic paradigms, but the technology is not infallible.Indiscriminate use of new technology will not improve health care but will only contribute to spiraling health care costs. The enhancement of clinical skills curricula must be accompanied by focusing clinical training back on patients and away from computerized data. In the words of Sir William Osler, "it is a safe rule to have no teaching without a patient for a text, and the best teaching is that taught by the patient himself."

**It is an -association of Professors of Medicine (APM) perspective;www.im.org/APM.

\section{References}

1. Omori DM, Wong RY, Antonelli MA, Hemmer PA. Introduction to clinical medicine: a time for consensus and integration. Am J Med 2005;118:189-94. 